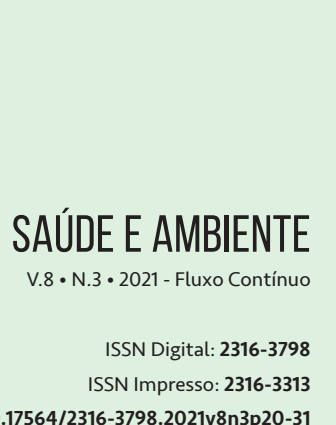

DOI: 10.17564/2316-3798.2021v8n3p20-31

\section{IMPACTO DA EXPLORAÇ̃̃O ARTESANAL DE OURO NA SAÚDE DOS GARIMPOS DO DISTRITO DE MANICA - MOÇAMBIQUE}

IMPACT OF GOLD CRAFTS EXPLORATION ON THE HEALTH OF PANNING DISTRICT OF MANICA - MOZAMBIQUE

\section{IMPACTO DE LA EXPLOTACIÓN DE ARTESANÍAS DE ORO EN LA SALUDDE LAS MINERÍAS DEL DISTRITO DE MANICA - MOZAMBIQUE}

\section{RESUMO}

Este pesquisa visa identificar os impactos da exploração artesanal de ouro na saúde dos garimpos do distrito de Manica - Moçambique. Para a sua materialização foi realizada a pesquisa bibliográfica, documental, observação, entrevista e inquérito aos garimpos e aos profissionais de saúde. Os resultados mostraram as doenças que afetam os garimpeiros estão relacionados com as condições em que a atividade mineira é realizada, isto mostra que existe deficiência no uso de equipamento de proteção individual e coletivo. Foram identificadas várias doenças oriundas da atividade garimpo, designadamente infeção das veias respiratórias superiores; feridas incisa; agressões; quedas, lombalgias; dores abdominais, tosse, dores de peito, filarias entre outras. Verificou-se que nas comunidades em estudo a dieta alimentar não é regular, que pode resultar no surgimento de doenças identificadas tais como estresse, depressão, alteração de humor, agressão entre outras. Este estudo evidencia a necessidade de realização de uma exploração sustentável do ouro de modo a garantir a continuidade da atividade de forma saudável sem deixar de lado a educação ambiental que possa promover mudança de comportamento por parte dos garimpeiros.

\section{PALAVRAS-CHAVE}

Saúde. Garimpo. Ouro. Manica. 


\section{ABSTRACT}

This research aims to identify the impacts of artisanal gold mining on the health of the mines in the district of Manica - Mozambique. For its materialization, bibliographical, documentary research, observation, interview and survey of garimpos and health professionals were carried out. The results showed the diseases that affect the prospectors are related to the conditions in which the mining activity is carried out, this shows that there is a deficiency in the use of individual and collective protective equipment. Several diseases have been identified as a result of the mining activity, namely infection of the upper respiratory veins; incised wounds; aggressions; falls, low back pain; abdominal pain, cough, chest pain, filarias and others. It was found that in the communities under study the diet is not regular, which can result in the emergence of identified diseases such as stress, depression, mood swings, aggression, among others. This study highlights the need to carry out a sustainable exploitation of gold in order to guarantee the continuity of the activity in a healthy way without neglecting environmental education that can promote behavior change on the part of the miners.

\section{KEYWORDS}

Health. Panning. Gold. Manica.

\section{RESUMEN}

Esta investigación tiene como objetivo identificar los impactos de la minería artesanal de oro en la salud de las minas en el distrito de Manica - Mozambique. Para su materialización se realizó investigación bibliográfica, documental, observación, entrevista y encuesta a las minerías y profesionales de la salud. Los resultados mostraron que las enfermedades que afectan a los garimpeiros están relacionadas con las condiciones en las que se desarrolla la actividad minera, esto demuestra que existe una deficiencia en el uso de equipos de protección individual y colectiva. Se han identificado varias enfermedades como resultado de la actividad minera, a saber, infección de las venas respiratorias superiores; heridas incisas; agresiones; caídas, lumbalgia; dolor abdominal, tos, dolor de pecho, filarias y otros. Se encontró que en las comunidades en estudio la dieta no es regular, lo que puede resultar en la aparición de enfermedades identificadas como estrés, depresión, cambios de humor, agresiones, entre otras. Este estudio destaca la necesidad de realizar una explotación sostenible del oro a fin de garantizar la continuidad de la actividad de manera saludable sin descuidar la educación ambiental que pueda promover un cambio de comportamiento por parte de los mineros. 


\section{PALABRAS CLAVE}

Salud. Minería. Oro. Manica.

\section{INTRODUCÇÃO}

A atividade de extração artesanal de ouro no Distrito de Manica, Moçambique, ocorre em uma região montanhosa, considerada parte do chamado "Cratão de Zimbabwe" com elevações que atingem de 1500 à 2000 metros, com um clima do tipo temperado húmido e precipitação que varia na ordem de 1000 à1020 mm.

Historicamente a extração artesanal de ouro no distrito de Manica é caracterizada por exploração de depósitos aluvionar, mas com a exaustão destes depósitos, os garimpos estão actualmente a extrair ouro de depósitos primários, em veios de quartzo. A rota de processamento de extração de ouro em veio de quartzo compreende a etapa da amalgamação, que consiste na agregação de minerais de ouro promovida por mercúrio. Para obtenção de ouro puro, a amálgama (ouro + mercúrio) é submetida a etapa de queima, caracterizado pela retirada de mercúrio sob altas temperaturas, para retirada de mercúrio na forma de vapor. Este processo causa exposição directa dos garimpos ao vapor de mercúrio e na exposição indirecta da população circunvizinha às áreas de extração artesanal de ouro.

Os garimpos artesanais podem ser considerados como o grupo populacional mais exposto ao mercúrio, e, como usualmente esse processo é realizado no interior de suas residências, próximos a familiares e outras pessoas, também podem ser consideradas populações expostas, tendo como principal via de exposição a inalação do vapor de mercúrio metálico (WHO, 2008).

O mercúrio metálico disponível no solo, resultante da atividade do garimpo, pode também sofrer um processo de metilação em sedimentos dos rios, contaminando os peixes e causando um perigo potencial de exposição ao metil-mercúrio para toda a população circunvizinha (CAMERA, 1996).

Segundo Camera (1996), a partir do processo de trabalho garimpo, pode-se categorizar os expostos em: i) população ocupacionalmente exposta ao mercúrio metálico, incluindo os garimpos que queimam ouro, garimpos próximos às áreas de queima e funcionários de lojas que comercializam o ouro; ii) população em geral exposta ao mercúrio metálico, ou seja, pessoas próximas ao locais de garimpo e às lojas que comercializam o ouro, e população em geral ou ocupacional potencialmente exposta ao metil-mercúrio, abrangendo os consumidores de peixes.

A absorção do vapor de mercúrio metálico ocorre principalmente pela via respiratória, por meio da inalação, sendo parte depositada em tecidos, onde se conjuga com grupamentos sulfidrilas de proteínas (GALVÃO; COREY, 1987). Segundo Foá (1986), a percentagem de retenção nos pulmões varia de 74 a $80 \%$.

0 mercúrio é levado dos pulmões pelo sangue e se distribui no organismo, acumulando-se nos rins, sistema nervoso central, fígado, medula óssea, vias aéreas superiores, pele, parede intestinal, glândulas salivares, coração, músculos e placenta (ZAVARIZ; GLINA, 1993). Podendo causar intoxica- 
ção aguda, com predominância dos sinais e sintomas respiratórios e intoxicações subagudas e crônicas, afetando o sistema nervoso, rins e pele (ATSDR, 1989).

Segundo Schvartsman (1982) existem quatro momentos extremamente perigosos em relação à exposição ocupacional ao mercúrio em mulheres: i) fase pré-implantação - em que pode ocorrer mutagênese química; ii) primeiro trimestre da gravidez - em que pode ocorrer teratogênese a nível de distúrbios neurológicos; iii) terceiro trimestre da gestação - em que podem ocorrer fetopatias: espasticidade, amaurose, manifestações neurológicas; iv) lactação - em que o mercúrio pode ser levado ao bebê via leite materno.

A eliminação de mercúrio no organismo é feita principalmente pelos rins. 0 mercúrio em baixas concentrações pode levar a uma intoxicação crônica. O quadro clássico atinge o aparelho gastrintestinal, o sistema nervoso e as funções psíquicas, cujas alterações variam de quadros leves a muito graves (ZAVARIZ; GLINA, 1993).

Segundo Zavariz e Glina (1993), estes quadros podem ser: i) gastrintestinal, este manifesta-se por lesões orais, estomacais e intestinais; ii) neurológico, que pode manifestar-se por tremores, alterações de sensibilidade dolorosa, térmica e táctil, alteração de reflexos, coordenação motora e parkinsonismo; iii) psíquico, que pode constituir-se por uma variedade de outras anormalidade como alterações de personalidade, denominada eretismo psíquico, com sinais típicos e precoces de intoxicação crônica por mercúrio, tais como: irritabilidade; alteração da sociabilidade; insônia; estado de ansiedade, timidez, labilidade emocional, e nos casos mais graves ocorre a diminuição da atenção, memória até um processo de despersonalização geral.

A atividade garimpo no Distrito de Manica é caracterizado por uso de técnicas e ferramentas rudimentares para concentração do ouro. Os vapores de mercúrio produzidos no processo de queima do amálgama para obtenção de ouro, bem como os restos do mercúrio presentes na lama, impregnada desse metal provocam sérios problemas ambientais e de saúde humana da população. As técnicas rudimentares utilizadas nestas minas artesanais remete-nos a uma reflexão em torno das consequências para a saúde e segurança dos garimpeiros e da população circunvizinha. Portanto, esta pesquisa objetivou estudar os impactos da exploração artesanal de ouro na saúde dos garimpos do distrito de Manica - Moçambique.

\section{METODOLOGIA}

Para o estudo, fez-se a observação directa das condições do trabalho, concretamente no que diz respeito ao uso de materiais de extração e processamento de ouro e equipamentos de proteção individual e coletiva. Fez-se levantamento das doenças diagnosticadas e registradas nos livros de registos de entradas e consultas externas no período entre 2019 e 2020, no Hospital Distrital de Manica e Posto de Saúde de Penhalonga, que atende a população das áreas da atividade do garimpo. Foram também realizadas as entrevistas dirigidas aos profissionais de saúde. Para obtenção de dados sobre saúde, segurança, doenças e acidentes de trabalho mais frequentes, foi aplicado questionário a um 
total de 30 garimpos. Estes questionários também foram aplicados as populações circunvizinhas das localidades de Mucrumadzi e Munhena, Moçambique.

O projeto foi aprovado pelo Serviço Distrital de Saúde Mulher e Acção Social de Manica e pela Diretoria do Hospital Distrital de Manica, Moçambique, respeitando a legislação local em relação aos aspectos éticos de coleta de dados e pesquisa com seres humanos.

\section{RESULTADOS E DISCUSSÃO}

\subsection{RESULTADOS OBTIDOS NO LIVRO DE REGISTRO DO HOSPITAL E POSTO DE SAÚDE}

\subsubsection{DOENCSAS RESPIRATÓRIA}

Os resultados da Figura 1, indicam que das doenças respiratorias diagnosticadas no Hospital Distrital de Manica e no Posto de Saúde de Penhalonga, designadamente: infecção das veias respiratorias superiores (IVRS), pneumonia, tosse, asma e brônquio pneumonia. As doencas mais infensiosas mais frequentes são infecção das vias respiratorias superiores (IVRS) e Pneumonia (PN), que correspondem $53,5 \%$ e $31,6 \%$, respectivamente. Este facto pode estar ligado a inalação de vapor de mercúrio resultante do processo de queima da amálgama e aerssóis resultante dos processo de lavra e britagem de sedimentos e rochas ricas em minerais de ouro, o que pode estar relacionado com o não uso de equipamento de protecção individial e utilização de técnicas redumentares de extração e processamento artesanal de ouro.

Os problemas de saúde humana causados pela inalacao do vapor de mercúrio acontece quando este é levado dos pulmões pelo sangue e se distribui no organismo, acumulando-se nos rins, sistema nervoso central, fígado, medula óssea, vias aéreas superiores, pele, parede intestinal, glândulas salivares, coração, músculos e placenta (ZAVARIZ; GLINA, 1993). A Agency for Toxic Substances and Disease Registry sustenta ainda que o mercúrio pode causar intoxicação aguda, com predominância dos sinais e sintomas respiratórios e intoxicações subagudas e crônicas, afetando o sistema nervoso, rins e pele (ATSDR, 1989). Alguns destes problemas de saúde podem ser visualizados na figura que ilustra os casos de doenças respiratórias diagnosticadas no hospital e posto de saúde dos locais de estudo. 
Figura 1 - Doenças respiratórias diagnosticadas no hospital e posto de saúde das áreas de atividade garimpo nos anos de 2019 e 2020, Distrito de Manica, Moçambique

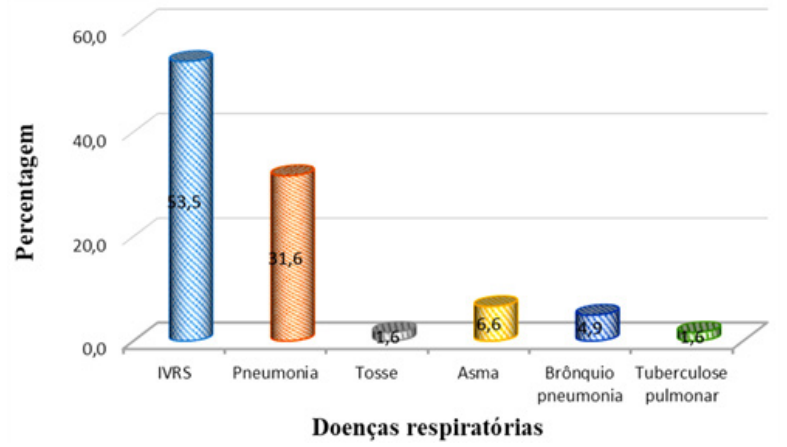

Fonte: Dados da pesquisa.

\subsubsection{DOENÇAS ERGONÓMICAS}

No que concerne aos resultados das doenças ergonómicas descrita na Figura 2, indicam que as doenças mais frequentes são a dor abdominal, feridas incisas e fraturas por quedas, que correspondem a 47,9\%, 17,1\% e 17,1\%, respetivamente. Acredita-se que a questão da dieta alimentar e segurança no trabalho, concretamente o não uso de equipamento de proteção individual e coletiva, e a má postura na realização das tarefas podem estar na origem destas doenças. Segundo Gonçalves (2012) a utilização destes equipamentos, pode marcar a diferença entre um trabalho em segurança ou um acidente de trabalho que pode impedir o mineiro de laborar durante várias semanas.

Muitos aspectos de trabalho em minas envolvem os riscos de lesões nos membros superiores e inferiores ou na coluna vertebral, quer devido as terefas de operação manual quer a postura incorrecta (JENININGS; WALLE, 2003).

Figura 2 - Doenças ergonómicas diagnosticadas no hospital e posto de saúde das áreas de atividade garimpo nos anos de 2019 e 2020, Distrito de Manica, Moçambique

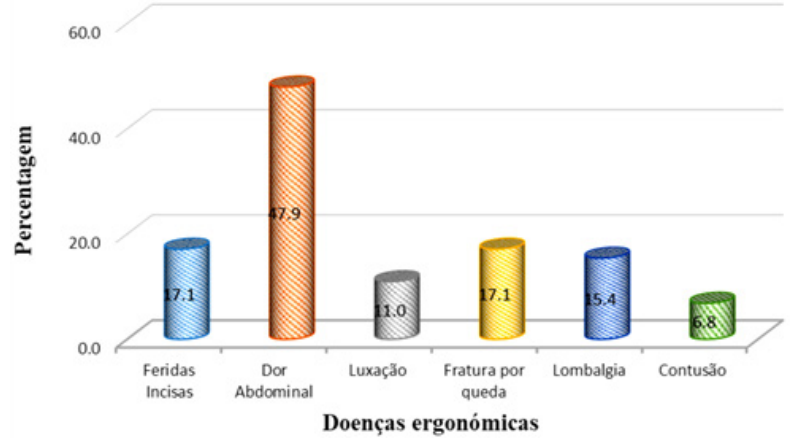

Fonte: Dados da pesquisa. 


\subsubsection{DOENÇAS PSICOLÓGICAS}

Os resultados das doenças psicológicas ilustrados na Figura 3, indicam a frequência de agressão, estresse, depressão, alteração de humor e alteração de personalidades e carácter nas áreas de atividade garimpo, sendo a agressão, estresse e alteração do humor casos mais predominantes quando analisado o grupo das doenças psicológicas, com 27,0\%, 22,2\% e 19,7\% respectivamente. Estas podem estar ligadas ao consumo de alcool e estupefacientes reportados por alguns membros da comunidade e também pelos enfermeiros, justificado pelos garimpeiros como sendo vias para alívio da dor e garantir maior resistencia para o trabalho, associado às comemorações quando o ouro é encontrado e vendido com sucesso. Estes casos podem também ser associados a situações de conflitos resultante de partilha de espaços para a prospecção, frustração pelo insucesso na busca do ouro e problemas na divisão dos lucros após a venda.

Há que frisar que os problemas apresentados, também podem estar associadas a intoxicação por mercúrio, como sustenta Zavaris e Glina (1992), ao afirmarem que quando inalado o mercúrio pode afetar o quadro psíquico, constituindo uma variedade de outras anormalidades como alterações de personalidade, denominada eretismo psíquico, com sinais típicos e precoces de intoxicação crônica por mercúrio, tais como: irritabilidade, alteração da sociabilidade, insônia, estado de ansiedade, timidez, labilidade emocional e nos casos mais graves ocorre a diminuição da atenção, memoria até um processo de despersonalização geral.

Há que realçar casos de problemas psicológicos como resultantes da ansiedade excessiva, da incapacidade de atingir seus objetivos que pode levar o individuo à estresse, depressão e/ou alterações da sua personalidade, afetando de certa forma as relações interpessoais.

Em relação ao estresse, é fundamental compreender como pode manifestar-se no organismo dos garimpeiros. Jeninings e Walle (2003), sugerem que os trabalhadores devem ser informados sobre a naturea do estresse do calor e seus efeitos adversos, assim como as medidas de proteção.

Figura 3 - Doenças psicológicas diagnosticadas no hospital e posto de saúde das áreas de atividade garimpo nos anos de 2019 e 2020, Distrito de Manica, Moçambique

40.0

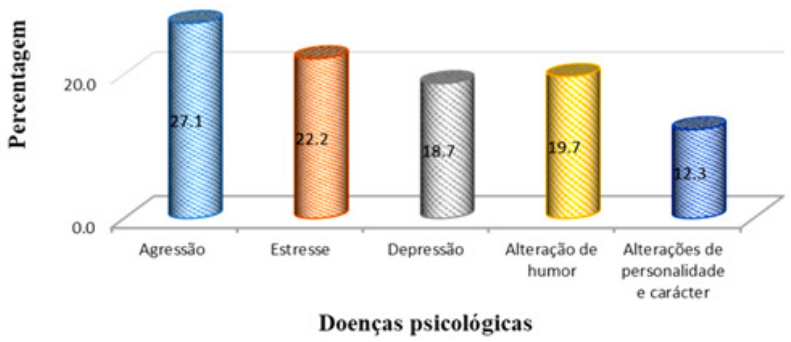

Fonte: Dados da pesquisa. 


\subsubsection{DOENÇAS INFECIOSAS}

Os resultados das doenças infeciosas ilustrados na Figura 4, indicam a maior frequência de malária, gastrite aguda e infeção renal aguda (IRA), com 84,7\%, 33,9\% e 11,3\%, respetivamente. Nesta listas, a malária constitui a doença infecciosa mais registada. Isto pode resultar da dificiencia de condições de saneamento existentes nas áreas de extração e processamento de ouro, observando-se águas estagnada usadas na etapa de processamento de ouro, o que constitui focos de multiplicação de mosquito, afectando não apenas os garimpeiros como também a comunidade circunvizinha.

Os casos de gastrite aguda podem ser explicados pelos hábitos alimentares adoptados não só pelos garimpeiros como também pela comunidade. Estes podem não estar a observar a regularidade nas dietas alimentares, existindo casos de uma refeicao ao dia. Para além da regularidade alimentar, observa-se também a ingestão de alimentos confenccionados de forma não adequadas.

Foram também observados, em pequena percentagem, casos de infeção urinária e de impotência sexual. Quanto a este último caso, os casos registrados oficialmente, podem não refletirem a realidade pelo facto da maioria não reportarem às unidades sanitárias estas doenças. Na entrevista aos garimpos, afirmaram que tem sido detectadas problemas relacionados com a infecção urinária, bem como situações relacionadas com a impotência sexual que é uma das consequências de inalação de mercúrio.

Figura 4 - Doenças infeciosas diagnosticadas no hospital e posto de saúde das áreas de atividade garimpo nos anos de 2019 e 2020, Distrito de Manica, Moçambique

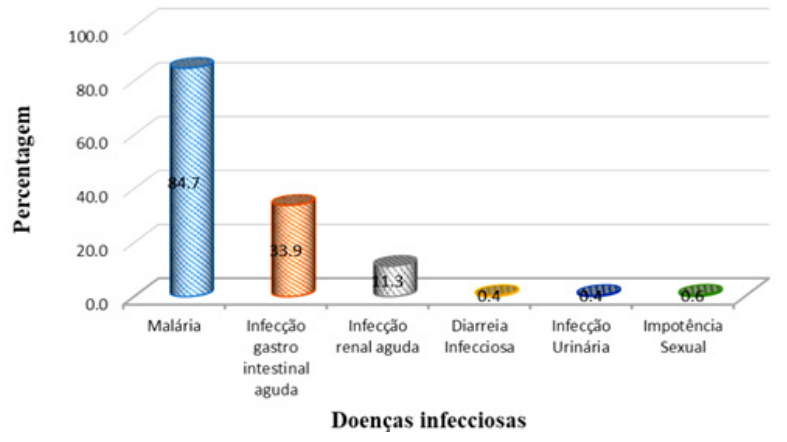

Fonte: Dados da pesquisa.

\subsection{RESULTADOS OBTIDOS COM BASE NO QUESTIONÁRIO E ENTREVISTA DIRIGIDAS AOS GARIMPOS}

\subsubsection{DOENÇAS MAIS FREQUENTES}

De acordo com a Figura 5, os resultados apontam que a tosse, dores no peito, filária e dores musculares são as mais frequentes de ocorrerem, com 83,3\%, 33,3\%, 23,3\% e 20\%, respetivamente. Estas doenças, pode-se justificar pelo facto de muitos deles não usarem equipamento de proteção, 
contribuindo desta forma para as doenças apresentadas. Para caso da filária, pode estar associada ao facto dos garimpos permanecerem por muito tempo com os pés mergulhados nas águas estagnadas das piscinas de lavagem de mercúrio. Há que destacar, também, a questão relacionada com a mudança de atitudes face a situações de risco, que pode estar associada a questão de défice de uma educação para a saúde (NASCIMENTO, 2019).

Figura 5 - Doenças frequentes nas áreas de atividade garimpo nos anos de 2019 e 2020, Distrito de Manica, Moçambique

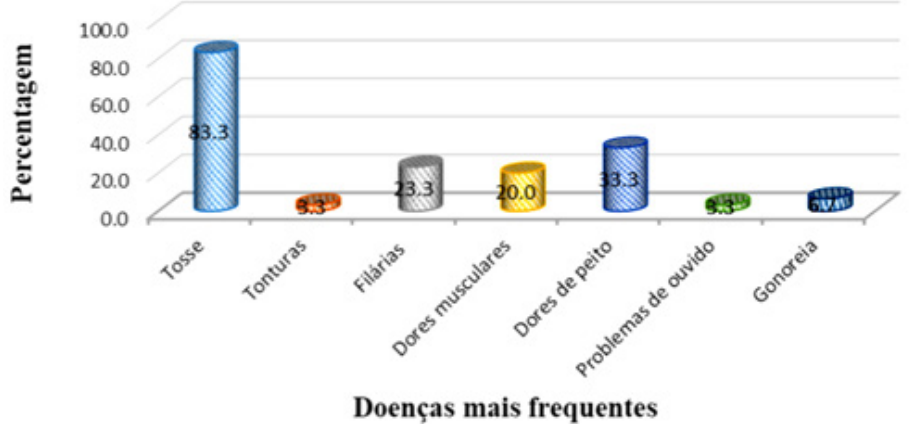

Fonte: Dados da pesquisa.

\subsubsection{EQUIPAMENTO DE SEGURANÇA}

No que concerne a questão relacionada com o uso de equipamento de seguranca no trabalho, o cinto de segurança e proteção dos ouvidos não são observados. Todavia, apesar de indicarem que as máscaras e as botas são usadas, não foi observado pelos pesquisadores a rigorosidade de seu uso, pois, que apenas um em cada 10 garimpos, apresentava-se de botas. No entanto, o não uso desses equipamentos, pode condicionar problemas de saúde anteriorimente referenciados, que estão ligados de forma directa ou indirecta com a activdade que exercem, como por exemplo, tosse, feridas enciza, filárias, entre outras doenças.

\subsubsection{ACIDENTE DE TRABALHO}

Quanto aos acidentes de trabalho, a intoxicação pelo mercúrio como sendo a mais frequente, seguida de quedas e queimaduras, quando esses exercem as atividades de extração e processamento de ouro. Portanto, esses resultados tornam-se preocupantes na medida em que a exposição de mercúrio no organismo humano, dá origem a vários problemas de saúde como sustentam Zavaris e Glina (1992). Esses resultados revelam a necessidade de promover a educação ligada a saúde, como afirma Nascimento (2019), as acções desenvolvidas no cuidado de saúde no contexto de garimpo, necessitam de atividades de educação em saude com vista a promover o autocuidado. 


\section{CONCLUSÃO}

Os problemas de saúde resultantes da exploração artesanal de ouro afetam não apenas aos garimpos como também as comunidades das áreas onde ocorrem a atividade de extração e processamento artesanal de ouro. Nesta atividade, verificou-se deficiência no uso de equipamento de proteção individual e coletiva pelos garimpos, factos que podem contribuírem na contração de doenças respiratórias, ergonómicas, psicológicas e infeciosas, verificadas. A dificuldade no manuseamento de equipamentos de operação, dieta alimentar, consumo de álcool e/ou estupefaciente, concorrem para a contração das doenças ergonómicas. Para além dos problemas resultantes da intoxicação por mercúrio, foi possível identificar os problemas de saúde oriundos do défice do saneamento, como é o caso da malária.

\section{AGRADECIMENTOS}

Os autores agradecem ao Fundo Nacional de Investigação - Moçambique pelo financiamento do projecto.

A todos que contribuíram para a realização das atividades, desde os técnicos e agricultores, vai o grande apreso de agradecimento.

\section{REFERÊNCIAS}

ATSDR - Agency for Toxic Substances and Disease Registry. Toxicological profile for mercury. Atlanta: GA. U.S. Public Health Service, 1989.

CÂMARA, V. M. Estudo dos níveis de exposição e efeitos à saúde por mercúrio metálico em uma população urbana de Poconé. Cad. Saúde Públ., Rio de Janeiro, v. 12, n.1, p. 69-77, 1996.

FOÁ, V., CAIMI, L. Patologia del mercúrio e suoi composti. Tratatto di Medicina del Lavoro (E. Sartorelli). Padova: Piccin, 1981. p. 353-376.

GALVÃO, L. A. C.; COREY, G. Mercurio. Serie Vigilancia 7, Metepec: Centro Panamericano de Ecología Humana y Salud, OPS/w, 1987.

GONÇALVES A. C. R. Riscos Associados a exploração mineira: caso das minas da Panasqueira. Cad Geograf, n. 30/31, p. 131-142, 2012.

JENININGS, N., WALLE, M. Segurança e saúde em minas de superfície de pequeno porte. Brasilia: Secretaria Internacional de trabalho, 2003. 
NASCIMENTO, V. F. et al. Vulnerabilidades em saúde de garimpeiros de uma região amazónica. Rev Enferm Actual, n. 37, p. 30-49, 2019.

SCHVARTSMAN, S. A gestante e a nutriz e a contaminação química do ambiente de trabalho. Rev Bras Saúde Ocup, v. 10, p. 86-90, 1982.

WHO - World Health Organization. Guidance for identifying populations at risk from mercury exposure. 2008. Disponível em: http://www.cetem.gov.br/publicacao/ extracao_de_ouro/ capitulo_11.pdf. Acesso em: 3 set. 2020.

ZAVARIZ, C.; GLINA, D. M. R. Efeitos da exposição ocupacional ao mercúrio em trabalhadores de uma indústria de lâmpadas elétricas localizada em Santo Amaro. Cad. Saúde Públ., v. 9, v. 2, p. 117-129, 1993. 
1 Graduado em Psicologia; Professor da Universidade Púnguè - Moçambique. E-mail: amunacachuma@yahoo.com.br

2 Doutor e Tecnologia dos Minerais e Meio Ambiente; Professor da Universidade Púnguè - Moçambique.

E-mail: rasoedsonfernandes@gmail.com

3 Mestra em Geografia; Cidade de Chimoio, Manica - Moçambique; Professora da Universidade Púnguè - Moçambique. E-mail: katia.gotine@gmail.com

4 Mestre em Engenharia Civil; Professor da Universidade Púnguè - Moçambique. E-mail: mmcachepa@yahoo.com.br

5 Mestra em Biotecnologia; Professora da Universidade Púnguè - Moçambique.

E-mail: omadacussengua@yahoo.com.br

6 Mestre em Auditoria e Gestão de Empresa; Professor da Universidade Católica - Moçambique.

E-mail: nicsonjonissene@gmail.com

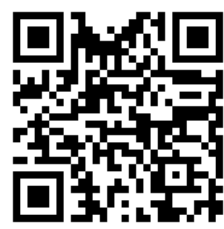

A autenticidade desse artigo pode ser conferida no site https://periodicos. set.edu.br

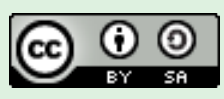

Este artigo é licenciado na modalidade acesso abertosob a Atribuição-Compartilhalgual CC BY-SA

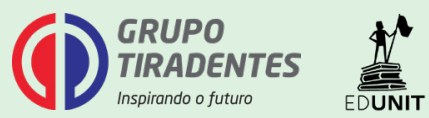

